\title{
ON THE APPROXIMATION OF FUNCTIONS BY MATRIX MEANS IN THE GENERALIZED HÖLDER METRIC
}

\author{
WŁODZIMIERZ ŁENSKI and BOGDAN SZAL \\ Faculty of Mathematics, Computer Science and Econometrics \\ University of Zielona Góra \\ Szafrana 4a, 65-516 Zielona Góra, Poland \\ E-mail:W.Lenski@wmie.uz.zgora.pl,B.Szal@wmie.uz.zgora.pl
}

\begin{abstract}
Under some assumptions on the matrix of a summability method, whose rows are sequences of bounded variation, we obtain a generalization and an improvement of some results of Xie-Hua Sun and L. Leindler.
\end{abstract}

1. Introduction. Let $f$ be a continuous and $2 \pi$-periodic function $\left(f \in C_{2 \pi}\right)$ and let

$$
f(x) \sim \frac{a_{0}}{2}+\sum_{n=1}^{\infty}\left(a_{n} \cos n x+b_{n} \sin n x\right)
$$

be its Fourier series. Denote by $S_{n}(x)=S_{n}(f, x)$ the $n$-th partial sum of (1.1) and by $\omega(f, \delta)$ the modulus of continuity of $f \in C_{2 \pi}$.

The usual supremum norm will be denoted by $\|\cdot\|_{C}$.

Let $\omega$ be a nondecreasing continuous function on the interval $[0,2 \pi]$ having the properties

$$
\omega(0)=0, \quad \omega\left(\delta_{1}+\delta_{2}\right) \leq \omega\left(\delta_{1}\right)+\omega\left(\delta_{2}\right) .
$$

Such a function will be called a modulus of continuity.

Denote by $H^{\omega}$ the class of functions

$$
H^{\omega}:=\left\{f \in C_{2 \pi} ;|f(x)-f(y)| \leq C \omega(|x-y|)\right\},
$$

where $C$ is a positive constant. For $f \in H^{\omega}$, we define the norm $\|\cdot\|_{\omega}$ by the formula

$$
\|f\|_{\omega}:=\|f\|_{C}+\sup _{x, y}\left|\Delta^{\omega} f(x, y)\right|
$$

2000 Mathematics Subject Classification: 42A24, 41A25.

Key words and phrases: trigonometric approximation, matrix means, special sequences.

The paper is in final form and no version of it will be published elsewhere. 
where

$$
\Delta^{\omega} f(x, y)=\frac{|f(x)-f(y)|}{\omega(|x-y|)}, \quad x \neq y .
$$

If $\omega(t)=C_{1}|t|^{\alpha}(0<\alpha \leq 1)$, where $C_{1}$ is a positive constant, then

$$
H^{\alpha}=\left\{f \in C_{2 \pi} ;|f(x)-f(y)| \leq C_{1}|x-y|^{\alpha}, 0<\alpha \leq 1\right\}
$$

is a Banach space and the metric induced by the norm $\|\cdot\|_{\alpha}$ on $H^{\alpha}$ is said to be the Hölder metric.

Let $A:=\left(a_{n k}\right)(k, n=0,1, \ldots)$ be a lower triangular infinite matrix of real numbers satisfying the following conditions:

$$
\text { i) } a_{n k} \geq 0(k=0,1, \ldots), a_{n k}=0,(k>n) \text { and } \sum_{k=0}^{n} a_{n k}=1,
$$

where $n=0,1,2, \ldots$,

$$
\text { ii) for every } k, a_{n k} \rightarrow 0(n \rightarrow \infty) \text {. }
$$

Let the $A$-transformation of $\left(S_{n}(f ; x)\right)$ be given by

$$
T_{n}(f):=T_{n}(f ; x):=\sum_{k=0}^{n} a_{n k} S_{k}(f ; x) \quad(n=0,1, \ldots) .
$$

and let

$$
A_{n k}=\sum_{r=n-k+1}^{n} a_{n r}, \quad(k=1,2, \ldots, n+1) .
$$

Let $\omega(t)$ be a given modulus of continuity satisfying the following condition:

$$
\frac{(\omega(f ; t))^{\frac{p}{q}}}{\omega(t)}=O(1) \quad\left(t \rightarrow 0_{+}\right),
$$

for $0 \leq p<q \leq 1$ and $f \in C$.

In [6] Xie-Hua Sun proved the following theorems:

THEOREM 1. Let $A=\left(a_{n k}\right)$ satisfy the conditions (1.2), (1.3) and $a_{n k} \leq a_{n k+1}$ for $k=0,1, \ldots, n-1$ and $n=0,1, \ldots$ If $f \in C$ and $\omega(f ; t), \omega(t)$ satisfy (1.4) then

$$
\left\|T_{n}(f)-f\right\|_{\omega}=O\left(\left\{\sum_{k=1}^{n} \frac{A_{n k}}{k}\right\}^{\frac{p}{q}}\left\{\sum_{k=1}^{n} A_{n k} \frac{\omega\left(f, \frac{1}{k}\right)}{k}\right\}^{1-\frac{p}{q}}\right) .
$$

THEOREM 2. Let $A=\left(a_{n k}\right)$ satisfy the conditions (1.2), (1.3) and $a_{n k} \geq a_{n k+1}$ for $k=0,1, \ldots, n-1$ and $n=0,1, \ldots$ If $f \in C$ and $\omega(f ; t), \omega(t)$ satisfy (1.4) then

$$
\left\|T_{n}(f)-f\right\|_{\omega}=O\left(\left\{\sum_{k=0}^{n} a_{n k} \omega\left(f, \frac{1}{k+1}\right)\right\}^{1-\frac{p}{q}}\right) .
$$

Now we define two classes of sequences ([3]).

A sequence $c:=\left(c_{n}\right)$ of nonnegative numbers tending to zero is called the Rest Bounded Variation Sequence, or briefly $c \in R B V S$, if it has the property

$$
\sum_{k=m}^{\infty}\left|c_{n}-c_{n+1}\right| \leq K(c) c_{m}
$$

for all natural numbers $m$, where $K(c)$ is a constant depending only on $c$. 
A sequence $c:=\left(c_{n}\right)$ of nonnegative numbers will be called the Head Bounded Variation Sequence, or briefly $c \in H B V S$, if it has the property

$$
\sum_{k=0}^{m-1}\left|c_{n}-c_{n+1}\right| \leq K(c) c_{m}
$$

for all natural numbers $m$, or only for all $m \leq N$ if the sequence $c$ has only finite nonzero terms and the last nonzero term is $c_{N}$.

Therefore we assume that the sequence $\left(K\left(\alpha_{n}\right)\right)_{n=0}^{\infty}$ is bounded, that is, that there exists a constant $K$ such that

$$
0 \leq K\left(\alpha_{n}\right) \leq K
$$

holds for all $n$, where $K\left(\alpha_{n}\right)$ denote the sequence of constants appearing in the inequalities (1.7) or (1.8) for the sequence $\alpha_{n}:=\left(a_{n k}\right)_{k=0}^{\infty}$. Now we can give the conditions to be used later on. We assume that for all $n$ and $0 \leq m \leq n$

$$
\sum_{k=m}^{\infty}\left|a_{n k}-a_{n k+1}\right| \leq K a_{n m}
$$

and

$$
\sum_{k=0}^{m-1}\left|a_{n k}-a_{n k+1}\right| \leq K a_{n m}
$$

hold if $\alpha_{n}:=\left(a_{n k}\right)_{k=0}^{\infty}$ belongs to $R B V S$ or $H B V S$, respectively.

In [3] L. Leindler proved the following theorem:

THEOREM 3. Let us assume that (1.2) and (1.9) hold. Then for $f \in C_{2 \pi}$

$$
\left\|T_{n}(f)-f\right\|_{C}=O\left(\omega\left(f ; \frac{\pi}{n}\right)+\sum_{k=1}^{n} \frac{\omega\left(f, \frac{\pi}{k}\right)}{k} \sum_{r=0}^{k+1} a_{n r}\right) .
$$

In the paper we present estimates of the deviation $T_{n}(f)-f$ in the norm $\|\cdot\|_{\omega}$ under more general assumptions on the elements of the matrix $A$.

Throughout the paper we shall use the following notations:

$$
\phi_{x}(t)=f(x+t)+f(x-t)-2 f(x), \quad A_{n}(u)=\sum_{k=0}^{n} a_{n k} \frac{\sin \left(k+\frac{1}{2}\right) u}{\sin \frac{1}{2} u} .
$$

By $K_{1}, K_{2}, \ldots$ we shall designate either an absolute constant or a constant depending on the indicated parameters, not necessarily the same at each occurrence.

2. Main results. Our main results are the following.

THEOREM 4. Let (1.2), (1.10) hold. If $f \in C$ and $\omega(f ; t), \omega(t)$ satisfy (1.4) then

$$
\left\|T_{n}(f)-f\right\|_{\omega}=O\left(\left\{\sum_{k=1}^{n+1} \frac{A_{n k}}{k}\right\}^{\frac{p}{q}}\left\{\sum_{k=1}^{n+1} A_{n k} \frac{\omega\left(f, \frac{1}{k}\right)}{k}\right\}^{1-\frac{p}{q}}\right) .
$$


Theorem 5. Let (1.2), (1.9) hold. If $f \in C$ and $\omega(f ; t), \omega(t)$ satisfy (1.4) then

$$
\left\|T_{n}(f)-f\right\|_{\omega}=O\left(\left\{\sum_{k=0}^{n} a_{n k} \omega\left(f, \frac{1}{k+1}\right)\right\}^{1-\frac{p}{q}}\right) .
$$

REMARK 1. If the elements of the matrix $A$ satisfy the condition $a_{n k} \leq a_{n k+1}$ for $k=$ $0,1, \ldots, n-1$ and $n=0,1, \ldots$, then the condition (1.10) also holds and therefore Theorem 1 is a corollary of Theorem 4. Analogously we can derive Theorem 2 from Theorem 5.

If in the assumptions of the Theorem 5 we take $\omega(|t|)=O\left(|t|^{p}\right)$ with $p=0$, then we have immediately the following corollary

COROLLARY 1. Under the assumptions of Theorem 3 we have

$$
\left\|T_{n}(f)-f\right\|_{C}=O\left(\sum_{k=0}^{n} a_{n k} \omega\left(f, \frac{1}{k+1}\right)\right) .
$$

REMARK 2. It is easy to see that from the above estimate the estimate (1.11) follows by $(1.9)$.

3. Lemmas. To prove our theorems we need the following lemmas.

LEMMA 1. If $\alpha_{n}:=\left(a_{n k}\right)_{k=0}^{\infty}$ belongs to $H B V S$, then for $\frac{1}{n} \leq u \leq \pi$

$$
\left|A_{n}(u)\right| \leq \frac{\pi^{2}\left(K\left(\alpha_{n}\right)+1\right)^{2}+\pi}{u} A_{n, u^{-1}},
$$

where $u^{-1}:=\max \left\{1,\left[u^{-1}\right]\right\}$.

Proof. An elementary calculation shows that

$$
\left|A_{n}(u)\right| \leq\left|\sum_{k=0}^{n-u^{-1}} a_{n k} \frac{\sin \left(k+\frac{1}{2}\right) u}{\sin \frac{1}{2} u}\right|+\frac{\pi}{u} \sum_{k=n-u^{-1}+1}^{n} a_{n k}=\left|\sum_{1}\right|+\frac{\pi}{u} A_{n, u^{-1}}
$$

Applying the Abel transformation and using (1.8), we get

$$
\begin{aligned}
\left|\sum_{1}\right| & \leq \sum_{k=0}^{n-u^{-1}-1}\left|a_{n k}-a_{n k+1}\right|\left(\frac{\sin \frac{k+1}{2} u}{\sin \frac{1}{2} u}\right)^{2}+a_{n, n-u^{-1}}\left(\frac{\sin \frac{n-u^{-1}+1}{2} u}{\sin \frac{1}{2} u}\right)^{2} \\
& \leq \frac{\pi^{2}}{u^{2}} \sum_{k=0}^{n-u^{-1}-1}\left|a_{n k}-a_{n k+1}\right|+\frac{\pi^{2}}{u^{2}} a_{n, n-u^{-1}} \leq \frac{\pi^{2}\left(K\left(\alpha_{n}\right)+1\right)}{u^{2}} a_{n, n-u^{-1}} .
\end{aligned}
$$

If $\alpha_{n}:=\left\{a_{n k}\right\}_{k=0}^{\infty}$ belongs to $H B V S$ then, by (1.8)

$$
a_{n \mu}-a_{n m} \leq\left|a_{n \mu}-a_{n m}\right| \leq \sum_{k=\mu}^{m-1}\left|a_{n k}-a_{n k+1}\right| \leq K\left(\alpha_{n}\right) a_{n m} .
$$

for any $m \geq \mu \geq 0$, whence we have

$$
a_{n \mu} \leq\left(K\left(\alpha_{n}\right)+1\right) a_{n m} .
$$

From this, we obtain

$$
\left|A_{n}(u)\right| \leq \frac{\pi^{2}\left(K\left(\alpha_{n}\right)+1\right)^{2}}{u} \sum_{k=n-u^{-1}+1}^{n} a_{n k}+\frac{\pi}{u} A_{n, u^{-1}}
$$




$$
\leq \frac{\pi^{2}\left(K\left(\alpha_{n}\right)+1\right)^{2}+\pi}{u} A_{n, u^{-1}}
$$

and our proof is complete.

LEMMA 2. If $\alpha_{n}:=\left(a_{n k}\right)_{k=0}^{\infty}$ belongs to $R B V S$, then for $f \in C_{2 \pi}$

$$
\left\|T_{n}(f)-f\right\|_{C} \leq 8\left(K\left(\alpha_{n}\right)+1\right)\left(2 K\left(\alpha_{n}\right)+1\right) \sum_{k=0}^{n} a_{n k} E_{k}(f),
$$

where $E_{n}(f)$ denotes the best approximation of the function $f$ by trigonometric polynomials of order at most $n$.

Proof. Let $m=m_{n} \geq 0$ be such that $2^{m} \leq n+1<2^{m+1}$ for $n=0,1, \ldots$, then applying the Abel transformation we get

$$
\begin{aligned}
& T_{n}(f ; x)-f(x)=\sum_{k=0}^{n} a_{n k}\left(S_{k}(f ; x)-f(x)\right)=a_{n 0}\left(S_{0}(f ; x)-f(x)\right) \\
& +\sum_{k=1}^{m-1} \sum_{i=2^{k-1}}^{2^{k}-1} a_{n i}\left(S_{i}(f ; x)-f(x)\right)+\sum_{k=2^{m-1}}^{n} a_{n k}\left(S_{k}(f ; x)-f(x)\right) \\
& =a_{n 0}\left(S_{0}(f ; x)-f(x)\right)+\sum_{k=1}^{m-1}\left(\sum_{i=2^{k-1}}^{2^{k}-2}\left(a_{n i}-a_{n i+1}\right) \sum_{l=2^{k-1}}^{i}\left(S_{l}(f ; x)-f(x)\right)\right. \\
& \left.+a_{n, 2^{k}-1} \sum_{i=2^{k-1}}^{2^{k}-1}\left(S_{i}(f ; x)-f(x)\right)\right) \\
& +\sum_{k=2^{m-1}}^{n-1}\left(a_{n k}-a_{n k+1}\right) \sum_{l=2^{m-1}}^{k}\left(S_{l}(f ; x)-f(x)\right)+a_{n n} \sum_{k=2^{m-1}}^{n}\left(S_{k}(f ; x)-f(x)\right) \\
& =a_{n 0}\left(\sigma_{00}(f ; x)-f(x)\right) \\
& +\sum_{k=1}^{m-1} \sum_{i=2^{k-1}}^{2^{k}-2}\left(\left(a_{n i}-a_{n i+1}\right)\left(i-2^{k-1}+1\right)\left(\sigma_{i, i-2^{k-1}}(f ; x)-f(x)\right)\right. \\
& \left.+a_{n, 2^{k}-1} 2^{k-1}\left(\sigma_{2^{k}-1,2^{k-1}-1}(f ; x)-f(x)\right)\right) \\
& +\sum_{k=2^{m-1}}^{n-1}\left(a_{n k}-a_{n k+1}\right)\left(k-2^{m-1}+1\right)\left(\sigma_{k, k-2^{m-1}}(f ; x)-f(x)\right) \\
& +a_{n n}\left(n-2^{m-1}+1\right)\left(\sigma_{n, n-2^{m-1}}(f ; x)-f(x)\right),
\end{aligned}
$$

where

$$
\sigma_{n, m}(f ; x)=\frac{1}{m+1} \sum_{k=n-m}^{n}\left(S_{k}(f ; x)-f(x)\right) \quad(0 \leq m \leq n)
$$

is the de la Vallée-Poussin mean. Using the well known inequality [6]

$$
\left\|\sigma_{n, m}(f)-f\right\|_{C} \leq 2 \frac{n+1}{m+1} E_{n-m}(f)
$$


we obtain

$$
\begin{gathered}
\left\|T_{n}(f)-f\right\|_{C} \leq 2 a_{n 0} E_{0}(f) \\
+\sum_{k=1}^{m-1}\left(2 E_{2^{k-1}}(f) \sum_{i=2^{k-1}}^{2^{k}-2}\left|a_{n i}-a_{n i+1}\right|(i+1)+4 a_{n, 2^{k}-1} 2^{k-1} E_{2^{k-1}}(f)\right) \\
+2 E_{2^{m-1}}(f) \sum_{k=2^{m-1}}^{n-1}\left|a_{n k}-a_{n k+1}\right|(k+1)+2 a_{n n}(n+1) E_{2^{m-1}}(f) .
\end{gathered}
$$

By (1.7) we have

$$
\begin{gathered}
\left\|T_{n}(f)-f\right\|_{C} \leq 2 a_{n 0} E_{0}(f) \\
+\sum_{k=1}^{m-1}\left(2 K\left(\alpha_{n}\right)\left(2^{k}-1\right) a_{n, 2^{k-1}} E_{2^{k-1}}(f)+4 a_{n, 2^{k}-1} 2^{k-1} E_{2^{k-1}}(f)\right) \\
+2 n K\left(\alpha_{n}\right) a_{n, 2^{m-1}} E_{2^{m-1}}(f)+2(n+1) a_{n n} E_{2^{m-1}}(f) .
\end{gathered}
$$

If $\alpha_{n}:=\left\{a_{n k}\right\}_{k=0}^{\infty}$ belongs to $R B V S$ then, by (1.7)

$$
\begin{gathered}
a_{n n}-a_{n m} \leq\left|a_{n m}-a_{n n}\right| \leq \sum_{k=m}^{n-1}\left|a_{n k}-a_{n k+1}\right| \\
\leq \sum_{k=m}^{\infty}\left|a_{n k}-a_{n k+1}\right| \leq K\left(\alpha_{n}\right) a_{n m}
\end{gathered}
$$

holds for any $n \geq m \geq 0$, whence we have

$$
a_{n n} \leq\left(K\left(\alpha_{n}\right)+1\right) a_{n m} .
$$

From this

$$
\begin{gathered}
\left\|T_{n}(f)-f\right\|_{C} \leq 2 a_{n 0} E_{0}(f) \\
+\left(2 K\left(\alpha_{n}\right)+1\right)\left(4 \sum_{k=1}^{m-1} a_{n, 2^{k-1}} 2^{k-1} E_{2^{k-1}}(f)+2(n+1) a_{n, 2^{m-1}} E_{2^{m-1}}(f)\right) .
\end{gathered}
$$

By (3.4)

$$
\begin{gathered}
(n+1) a_{n, 2^{m-1}} E_{2^{m-1}}(f) \leq 2^{m+1} a_{n, 2^{m-1}} E_{2^{m-1}}(f) \\
\leq 4 a_{n, 2^{m-1}} \sum_{k=2^{m-2}+1}^{2^{m-1}} E_{k}(f) \leq 4\left(K\left(\alpha_{n}\right)+1\right) \sum_{k=2^{m-2}+1}^{2^{m-1}} a_{n k} E_{k}(f)
\end{gathered}
$$

and for $k \geq 2$

$$
a_{n, 2^{k-1}} 2^{k-1} E_{2^{k-1}}(f) \leq 2 a_{n, 2^{k-1}} \sum_{i=2^{k-2}+1}^{2^{k-1}} E_{i}(f) \leq 2\left(K\left(\alpha_{n}\right)+1\right) \sum_{i=2^{k-2}+1}^{2^{k-1}} a_{n i} E_{i}(f) .
$$

Therefore

$$
\begin{gathered}
\left\|T_{n}(f)-f\right\|_{C} \leq 2 a_{n 0} E_{0}(f)+4\left(2 K\left(\alpha_{n}\right)+1\right) a_{n 1} E_{1}(f) \\
+8\left(K\left(\alpha_{n}\right)+1\right)\left(2 K\left(\alpha_{n}\right)+1\right)\left(\sum_{k=2}^{m-1} \sum_{i=2^{k-2}+1}^{2^{k-1}} a_{n i} E_{i}(f)+\sum_{k=2^{m-2}+1}^{2^{m-1}} a_{n k} E_{k}(f)\right)
\end{gathered}
$$




$$
\begin{aligned}
& \leq 8\left(K\left(\alpha_{n}\right)+1\right)\left(2 K\left(\alpha_{n}\right)+1\right) \sum_{k=0}^{2^{m-1}} a_{n k} E_{k}(f) \\
& \leq 8\left(K\left(\alpha_{n}\right)+1\right)\left(2 K\left(\alpha_{n}\right)+1\right) \sum_{k=0}^{n} a_{n k} E_{k}(f)
\end{aligned}
$$

and our proof is complete.

LEMMA 3. If $\alpha_{n}:=\left(a_{n k}\right)_{k=0}^{\infty}$ belongs to $R B V S$, then

$$
\int_{0}^{\pi}\left|A_{n}(t)\right| d t \leq 4 K\left(\alpha_{n}\right)\left(K\left(\alpha_{n}\right)+1\right) .
$$

Proof. Let $m=m_{n} \geq 0$ be such that $2^{m} \leq n+1<2^{m+1}$ for $n=0,1, \ldots$, then applying the Abel transformation we get

$$
\begin{gathered}
A_{n}(t)=\sum_{k=0}^{n}\left(a_{n k}-a_{n k+1}\right)(k+1) F_{k}(t)=\left(a_{n 0}-a_{n 1}\right) F_{0}(t) \\
+\sum_{k=1}^{m-1} \sum_{l=2^{k-1}}^{2^{k}-1}\left(a_{n l}-a_{n l+1}\right)(l+1) F_{l}(t)+(n+1) F_{n}(t) \sum_{k=2^{m-1}}^{n}\left(a_{n k}-a_{n k+1}\right),
\end{gathered}
$$

where $F_{n}(t)$ is the Fejér kernel. Since the kernel $F_{n}(t)$ is positive, using $(1.7)$ we can show that

$$
\begin{aligned}
\int_{0}^{\pi}\left|A_{n}(t)\right| d t \leq & \int_{0}^{\pi}\left\{\left|a_{n 0}-a_{n 1}\right| F_{0}(t)+\sum_{k=1}^{m-1} \sum_{l=2^{k-1}}^{2^{k}-1}\left|a_{n l}-a_{n l+1}\right|(l+1) F_{l}(t)\right. \\
& \left.+(n+1) F_{n}(t) \sum_{k=2^{m-1}}^{n}\left|a_{n k}-a_{n k+1}\right|\right\} d t \\
=\left|a_{n 0}-a_{n 1}\right| \int_{0}^{\pi} F_{0}(t) d t+\sum_{k=1}^{m-1} \sum_{l=2^{k-1}}^{2^{k}-1}\left|a_{n l}-a_{n l+1}\right|(l+1) \int_{0}^{\pi} F_{l}(t) d t & \\
& +(n+1) \sum_{k=2^{m-1}}^{n}\left|a_{n k}-a_{n k+1}\right| \int_{0}^{\pi} F_{n}(t) d t \\
= & \frac{1}{2}\left\{\left|a_{n 0}-a_{n 1}\right|+\sum_{k=1}^{m-1} \sum_{l=2^{k-1}}^{2^{k}-1}\left|a_{n l}-a_{n l+1}\right|(l+1)\right. \\
\leq & \frac{K\left(\alpha_{n}\right)}{2}\left(a_{n 0}+\sum_{k=1}^{m} 2^{k} a_{n, 2^{k-1}}+(n+1) a_{n, 2^{m-1}}\right) .
\end{aligned}
$$

By (3.4)

$$
(n+1) a_{n, 2^{m-1}} \leq 2^{m+1} a_{n, 2^{m-1}} \leq 8\left(K\left(\alpha_{n}\right)+1\right) \sum_{k=2^{m-2}+1}^{2^{m-1}} a_{n k}
$$


and for $k \geq 2$

$$
2^{k} a_{n, 2^{k-1}} \leq 4\left(K\left(\alpha_{n}\right)+1\right) \sum_{l=2^{k-2}+1}^{2^{k-1}} a_{n l} .
$$

Thus we obtain the desired estimate

$$
\begin{gathered}
\int_{0}^{\pi}\left|A_{n}(t)\right| d t \leq \frac{K\left(\alpha_{n}\right)}{2}\left(a_{n 0}+2 a_{n 1}+4\left(K\left(\alpha_{n}\right)+1\right) \sum_{k=1}^{m-1} \sum_{l=2^{k-2}+1}^{2^{k-1}} a_{n l}\right. \\
\left.+8\left(K\left(\alpha_{n}\right)+1\right) \sum_{k=2^{m-2}+1}^{2^{m-1}} a_{n k}\right) \\
\leq 4 K\left(\alpha_{n}\right)\left(K\left(\alpha_{n}\right)+1\right) \sum_{k=0}^{2^{m-1}} a_{n k} \\
\leq 4 K\left(\alpha_{n}\right)\left(K\left(\alpha_{n}\right)+1\right) \sum_{k=0}^{n} a_{n k}=4 K\left(\alpha_{n}\right)\left(K\left(\alpha_{n}\right)+1\right) .
\end{gathered}
$$

4. Proofs of the theorems. In this section we shall prove our theorems.

4.1. Proof of Theorem 4. Setting

$$
R_{n}(x, y)=R_{n}(x)-R_{n}(y)=\frac{1}{2 \pi} \int_{0}^{\pi}\left(\phi_{x}(t)-\phi_{y}(t)\right) A_{n}(t) d t
$$

and

$$
R_{n}(x)=T_{n}(f ; x)-f(x)=\frac{1}{2 \pi} \int_{0}^{\pi} \phi_{x}(t) A_{n}(t) d t
$$

we get

$$
\left|R_{n}(x, y)\right| \leq \frac{1}{2 \pi} \int_{0}^{\pi}\left|\phi_{x}(t)-\phi_{y}(t)\right|\left|A_{n}(t)\right| d t
$$

and

$$
\left|R_{n}(x)\right| \leq \frac{1}{2 \pi} \int_{0}^{\pi}\left|\phi_{x}(t)\right|\left|A_{n}(t)\right| d t \leq \frac{1}{\pi} \int_{0}^{\pi} \omega(f ; t)\left|A_{n}(t)\right| d t .
$$

It is clear that

$$
\left|\phi_{x}(t)-\phi_{y}(t)\right| \leq 4 \omega(f ;|x-y|)
$$

and

$$
\left|\phi_{x}(t)-\phi_{y}(t)\right| \leq 4 \omega(f ;|t|) .
$$

Hence, using (4.1), we have

$$
\begin{aligned}
\left|R_{n}(x, y)\right| \leq & \frac{2 C}{\pi} \omega(f ;|x-y|)\left(\int_{0}^{\frac{\pi}{n}}+\int_{\frac{\pi}{n}}^{\pi}\right)\left|A_{n}(t)\right| d t \\
& =\frac{2 C}{\pi} \omega(f ;|x-y|)\left(I_{1}+I_{2}\right) .
\end{aligned}
$$


It is obvious that

$$
I_{1} \leq \int_{0}^{\frac{\pi}{n}} \frac{1}{\sin \frac{1}{2} t} \sum_{k=0}^{n} a_{n k}\left|\sin \left(k+\frac{1}{2}\right) t\right| d t \leq(2 n+1) \frac{\pi}{n}<3 \pi .
$$

Using (3.1), we get

$$
\begin{gathered}
I_{2} \leq K_{1} \int_{\frac{\pi}{n}}^{\pi} \frac{A_{n, t-1}}{t} d t=K_{1} \int_{\frac{1}{\pi}}^{\frac{n}{\pi}} \frac{A_{n, t}}{t} d t \\
=K_{1} \sum_{k=1}^{n-1} \int_{\frac{k}{\pi}}^{\frac{k+1}{\pi}} \frac{A_{n, t}}{t} d t \leq K_{1} \sum_{k=1}^{n-1} \frac{A_{n, k+1}}{k} \\
\quad \leq 2 K_{1} \sum_{k=2}^{n} \frac{A_{n, k}}{k} \leq 2 K_{1} \sum_{k=1}^{n+1} \frac{A_{n, k}}{k} .
\end{gathered}
$$

From (3.2) and (1.2) we can observe that

$$
\sum_{k=1}^{n+1} \frac{A_{n, k}}{k}=\sum_{k=1}^{n+1} \frac{1}{k} \sum_{r=n-k+1}^{n} a_{n r} \geq \frac{1}{K+1} \sum_{k=1}^{n+1} a_{n, n-k+1}=\frac{1}{K+1} \sum_{k=0}^{n} a_{n k}=\frac{1}{K+1}
$$

and by (4.3)-(4.5), we obtain

$$
\left|R_{n}(x, y)\right| \leq K_{2} \omega(f ;|x-y|) \sum_{k=1}^{n+1} \frac{A_{n, k}}{k} .
$$

On the other hand, using (4.2), we have

$$
\begin{gathered}
\left|R_{n}(x, y)\right| \leq \frac{2}{\pi} \int_{0}^{\pi} \omega(f ; t)\left|A_{n}(t)\right| d t \\
=\frac{2}{\pi}\left(\int_{0}^{\frac{\pi}{n}}+\int_{\frac{\pi}{n}}^{\pi}\right) \omega(f ; t)\left|A_{n}(t)\right| d t=\frac{2}{\pi}\left(I_{1}^{\prime}+I_{2}^{\prime}\right) .
\end{gathered}
$$

Again by (1.2)

$$
I_{1}^{\prime} \leq \omega\left(f, \frac{\pi}{n}\right) \int_{0}^{\frac{\pi}{n}}\left|A_{n}(t)\right| d t \leq 3 \pi \omega\left(f, \frac{\pi}{n}\right) .
$$

and by (3.1) and using the monotonicity of the modulus of continuity

$$
\begin{aligned}
I_{2}^{\prime} & \leq K_{1} \int_{\frac{\pi}{n}}^{\pi} \omega(f ; t) \frac{A_{n, t^{-1}}}{t} d t=K_{1} \int_{\frac{1}{\pi}}^{\frac{n}{\pi}} \omega\left(f ; \frac{1}{t}\right) \frac{A_{n, t}}{t} d t \\
= & K_{1} \sum_{k=1}^{n-1} \int_{\frac{k}{\pi}}^{\frac{k+1}{\pi}} \omega\left(f ; \frac{1}{t}\right) \frac{A_{n, t}}{t} d t \leq K_{1} \sum_{k=1}^{n-1} \omega\left(f ; \frac{\pi}{k}\right) \frac{A_{n, k+1}}{k} \\
& \leq 8 K_{1} \sum_{k=2}^{n} \omega\left(f ; \frac{\pi}{k}\right) \frac{A_{n, k}}{k} \leq 8 K_{1} \sum_{k=1}^{n+1} \omega\left(f ; \frac{\pi}{k}\right) \frac{A_{n, k}}{k} .
\end{aligned}
$$

From the monotonicity of the modulus of continuity by (3.2) and (1.2)

$$
\sum_{k=1}^{n+1} \omega\left(f ; \frac{\pi}{k}\right) \frac{A_{n, k}}{k} \geq \omega\left(f, \frac{\pi}{n+1}\right) \sum_{k=1}^{n+1} \frac{A_{n, k}}{k}
$$




$$
\geq \frac{1}{2} \omega\left(f, \frac{\pi}{n}\right) \frac{1}{K+1} \sum_{k=0}^{n} a_{n k}=\frac{1}{2(K+1)} \omega\left(f, \frac{\pi}{n}\right) .
$$

Combining (4.6)-(4.9) we obtain

$$
\left|R_{n}(x, y)\right| \leq K_{3} \sum_{k=1}^{n+1} \omega\left(f ; \frac{\pi}{k}\right) \frac{A_{n, k}}{k}
$$

and the same estimate for $\left|R_{n}(x)\right|$ is true

$$
\left|R_{n}(x)\right| \leq K_{4} \sum_{k=1}^{n+1} \omega\left(f ; \frac{\pi}{k}\right) \frac{A_{n, k}}{k} .
$$

Using (4.6), (1.4) and (4.10), we get

$$
\begin{gathered}
\sup _{x, y}\left\{\Delta^{\omega} R_{n}(x, y)\right\}=\sup _{x, y}\left\{\frac{\left|R_{n}(x, y)\right|^{\frac{p}{q}}}{\omega(|x-y|)}\left|R_{n}(x, y)\right|^{1-\frac{p}{q}}\right\} \\
\leq K_{5}\left\{\sum_{k=1}^{n+1} \frac{A_{n k}}{k}\right\}^{\frac{p}{q}}\left\{\sum_{k=1}^{n+1} A_{n k} \frac{\omega\left(f, \frac{1}{k}\right)}{k}\right\}^{1-\frac{p}{q}} .
\end{gathered}
$$

Now collecting our partial results (4.11) and (4.12) we obtain (2.1), and this completes the proof.

4.2. Proof of Theorem 5. Using the same notations as in the above proof, from (4.1) and (3.5) we get

$$
\begin{gathered}
\left|R_{n}(x, y)\right| \leq \frac{2 C}{\pi} \omega(f ;|x-y|) \int_{0}^{\pi}\left|A_{n}(t)\right| d t \\
\leq \frac{8 C K(K+1)}{\pi} \omega(f ;|x-y|) .
\end{gathered}
$$

On the other hand

$$
\left|R_{n}(x, y)\right| \leq\left|T_{n}(f ; x)-f(x)\right|+\left|T_{n}(f ; y)-f(y)\right|
$$

by (4.2) and (3.3), whence we have

$$
\left|R_{n}(x, y)\right| \leq 16(K+1)(2 K+1) \sum_{k=0}^{n} a_{n k} E_{k}(f) \leq K_{1} \sum_{k=0}^{n} a_{n k} \omega\left(f, \frac{1}{k+1}\right) .
$$

Analogously, we can show that

$$
\begin{gathered}
\left|R_{n}(x)\right|=\left|T_{n}(f ; x)-f(x)\right| \leq 8(K+1)(2 K+1) \sum_{k=0}^{n} a_{n k} E_{k}(f) \\
\leq K_{2} \sum_{k=0}^{n} a_{n k} \omega\left(f, \frac{1}{k+1}\right) .
\end{gathered}
$$

Finally, using the same method as in the proof of Theorem 4, (2.2) follows from (4.13)(4.15). 


\section{References}

[1] P. Chandra, On the degree of approximation of a class of functions by means of Fourier series, Acta Math. Hungar. 52 (1988), 199-205.

[2] P. Chandra, A note on the degree of approximation of continuous function, Acta Math. Hungar. 62 (1993), 21-23.

[3] L. Leindler, On the degree of approximation of continuous functions, Acta Math. Hungar. 104 (2004), 105-113.

[4] R. N. Mohapatra and P. Chandra, Degree of approximation of functions in the Hölder metric, Acta Math. Hungar. 41 (1983), 67-76.

[5] T. Singh, Degree of approximation to functions in a normed spaces, Publ. Math. Debrecen 40 (1992), 261-271.

[6] X.-H. Sun, Degree of approximation of functions in the generalized Hölder metric, Indian J. Pure Appl. Math. 27 (1996), 407-417.

[7] Ch. J. de la Vallée-Poussin, Leçons sur l'approximation des fonctions d'une variable réelle, Paris, 1919. 
